\title{
Review of: "Galvanic corrosion inhibition from aspect of bonding orbital theory in $\mathrm{Cu} / \mathrm{Ru}$ barrier CMP"
}

\author{
Ali Davoodi ${ }^{1}$ \\ 1 Ferdowsi University of Mashhad
}

Potential competing interests: The author(s) declared that no potential competing interests exist.

In this research, galvanic corrosion in $\mathrm{Cu} / \mathrm{Ru}$ bimetallic system (used in semiconductor manufacturing process) was studied. In addition to the experiments, a theoretical approach was used for illustration of existed difference ( $\mathrm{Cu}$ is weaker than Ru regarding to corrosion). Furthermore, a pyridine carboxylic acid groups compound (nicotinic acid) was used for corrosion control. The results clearly shows that adding sufficient amount of nicotinic acid could reduce Cu potential gap compared to Ru film and therefore enhance the $\mathrm{Cu}$ corrosion resistance, leading to suppress the galvanic corrosion of $\mathrm{Cu}$ in $\mathrm{Cu} / \mathrm{Ru}$ couples.

1. Additional statistical evaluation like PSD (Power Spectral Density) analysis of of surface topography results could improve the reliability of surface roughness information in AFM images in Fig. 5. 